\title{
Leiomyosarcoma of the splenic vein: A case report
}

\author{
WENFANG WU ${ }^{1}$, XINXIANG ZHAO ${ }^{1}$, YAN WANG ${ }^{2}$, CONG DI $^{1}$, RENHUI CAI ${ }^{1}$, \\ YINGXUE ZHANG $^{1}$, SIYING CHEN ${ }^{1}$, WEI ZHANG ${ }^{1}$ and XIN YUE ${ }^{1}$ \\ Departments of ${ }^{1}$ Radiology and ${ }^{2}$ Pathology, The Second Affiliated Hospital of \\ Kunming Medical University, Kunming, Yunnan 650101, P.R. China
}

Received November 26, 2015; Accepted February 23, 2017

DOI: $10.3892 / \mathrm{ol} .2017 .6225$

\begin{abstract}
Leiomyosarcomas (LMSs) originating from the wall of blood vessels are aggressive and rare neoplasms. The current study describes a case of a 52-year-old man who presented with intermittent abdominal pain without weight loss or diarrhea. Computed tomography of the abdomen identified a $4-\mathrm{cm}$, solid, heterogeneous tumor in the tail of the pancreas, while a hypodense lesion was also noted in the right hepatic lobe. The patient subsequently underwent splenic pedicle tumor resection, splenectomy and liver tumor resection. Notably, immunohistochemical and histological analyses identified LMS, which had originated from the smooth muscle of the splenic vein. Currently available information regarding LMSs of the splenic vein and their management is also discussed, with the aim of improving diagnostic accuracy.
\end{abstract}

\section{Introduction}

Primary leiomyosarcoma (LMS) of the splenic vein is a rare malignant tumor and there have only been three previously reported cases $(1,2)$. Due to the rarity of the tumor, its imaging features have not yet been described in detail. Venous LMSs predominately occur in middle-aged women in the fifth and sixth decades of life (3). Clinical manifestations depend on the tumor position in the vessel wall (4). The symptoms of LMS include abdominal masses, abdominal pain, nausea and fever (5). The current study presents a case report on the magnetic resonance imaging (MRI) features of primary LMS of the splenic vein. A solid, heterogeneous mass was located in the tail of the pancreas. In portal phase, the splenic vein was embedded in the mass on T1-weighted imaging (T1WI). The patient underwent splenic pedicle tumor resection, splenectomy and liver tumor resection with no postoperative complications.

Correspondence to: Professor Xinxiang Zhao, Department of Radiology, The Second Affiliated Hospital of Kunming Medical University, 374 Dianmian Street, Kunming, Yunnan 650101, P.R. China

E-mail: 18314513590@163.com

Key words: leiomyosarcoma, splenic vein, splenectomy, magnetic resonance imaging, computed tomography
There is a limited amount of evidence demonstrating increased survival following adjuvant radiation, combination radiation and chemotherapy, in addition to surgical resection (6). Further information regarding the prognosis of the present patient is unavailable, as the patient did not undergo further adjuvant therapy or follow-up, and unique treatment recommendations or outcome data for such lesions remains to be established.

\section{Case report}

In June 2013, a 52-year-old male was admitted to the Gastroenterology Department of The Second Affiliated Hospital of Kunming Medical University (Kunming, China) presenting with a long history ( $>1$ year) of intermittent epigastric pain. The pain presented as either severe colic lasting for 20-30 min or as dull pain, which would be relieved following squatting. The medical history of the patient was unremarkable. A whole body positron emission tomography scan was performed at Yuxi People's Hospital (Yuxi, China) and revealed an irregular mass in the splenic hilum without increased metabolism.

An abdominal computed tomography (CT) scan performed at the Department of Radiology, The Second Affiliated Hospital of Kunming Medical University identified an irregular, hypodense mass measuring $\sim 4.1 \times 3.0 \mathrm{~cm}$ in the tail of the pancreas, while a hypodense lesion was also observed in the right hepatic lobe (Fig. 1). Following administration of contrast agent, lesions exhibited peripheral enhancement and there was a low-density zone in the center of the lesion without enhancement (Figs. 2 and 3). An MRI scan identified a solid, heterogeneous mass, which had fused with two nodules measuring $1.87 \times 2.44 \mathrm{~cm}$ and $2.46 \times 2.34 \mathrm{~cm}$, located in the tail of the pancreas. T1WI (Fig. 4) demonstrated slight hypointensity, while T2-WI exhibited slight hyperintensity (Fig. 5). Mild inhomogeneous enhancement was observed in the arterial phase (Fig. 6), strengthening in the portal phase (Fig. 7) was higher than the arterial phase and the splenic vein was embedded in the mass on T1WI enhanced images. The delay period exhibited inhomogeneous moderate enhancement (Fig. 8) and a small nodule was located between the liver VI (right hepatic lobe lower section) and the liver V (lower right anterior lobe of the liver). The nodule in the liver exhibited slight hypointensity on T1WI, which was similar to the liver signal on T2WI, and the lesion demonstrated peripheral enhancement post-contrast. 


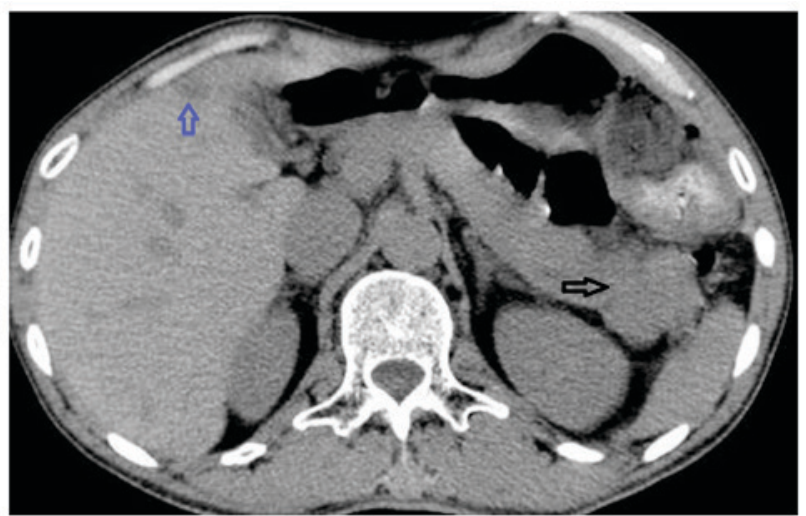

Figure 1. Abdominal computed tomography showed an irregular, hypodense mass measuring $4.1 \times 3.0 \mathrm{~cm}$ in the tail of the pancreas (black arrow), while a hypodense lesion was also observed in the right hepatic lobe (blue arrow).

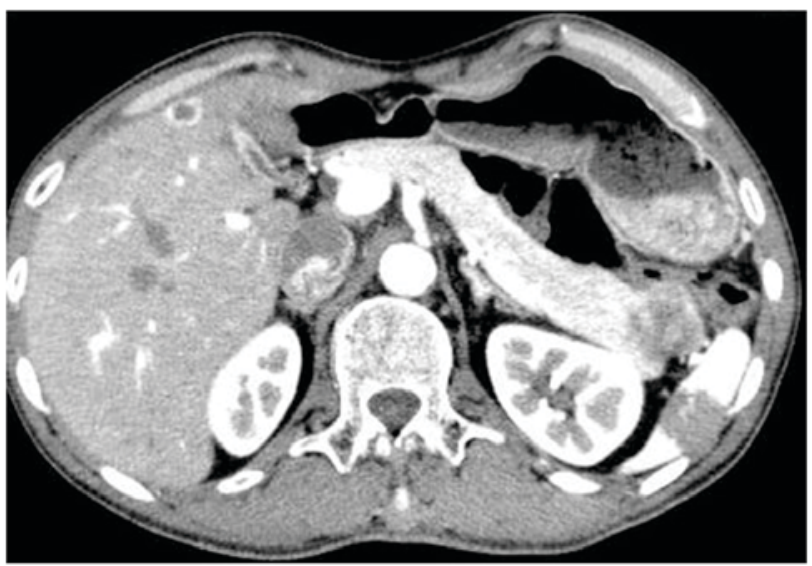

Figure 2. Post-contrast lesions in the tail of the pancreas exhibited peripheral enhancement. The nodule in the liver demonstrated peripheral enhancement.

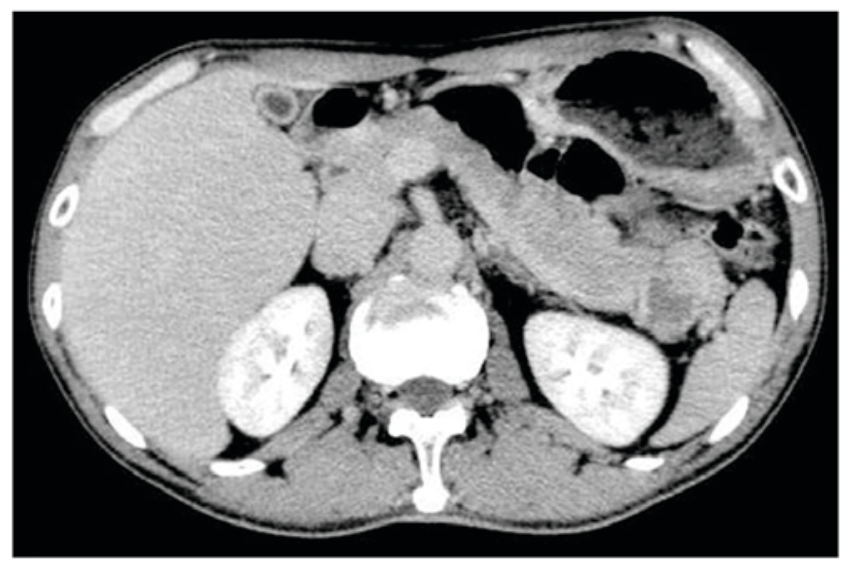

Figure 3. Post-contrast image demonstrating a low-density zone in the center of the lesion (located in the tail of the pancreas) without enhancement.

An initial diagnosis of pancreatic cancer was considered and surgery was scheduled. The patient subsequently underwent splenic pedicle tumor resection, splenectomy and liver tumor resection. Following surgical resection, specimens were fixed in $10 \%$ neutral formalin for $12 \mathrm{~h}$, embedded in paraffin and cut into $4 \mu \mathrm{m}$-thick sections. These sections

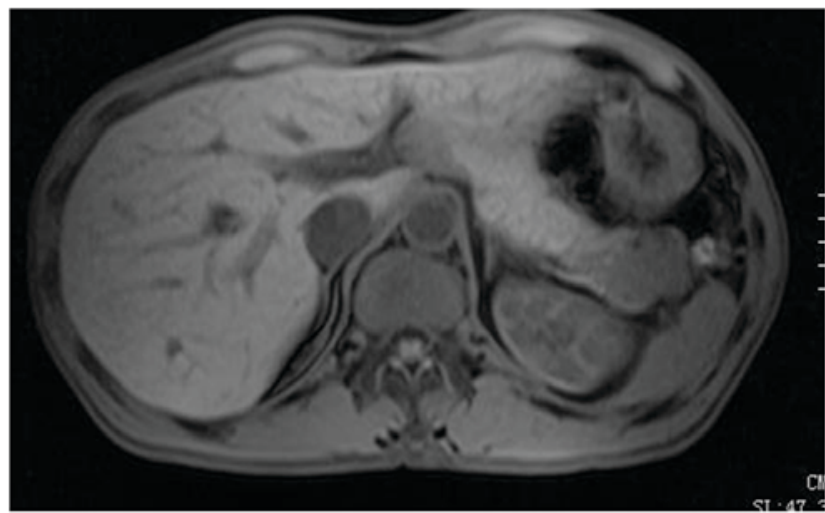

Figure 4. A magnetic resonance image showing a solid, heterogeneous mass located in the tail of the pancreas. The lesion demonstrated slight hypointensity on T1-weighted images.

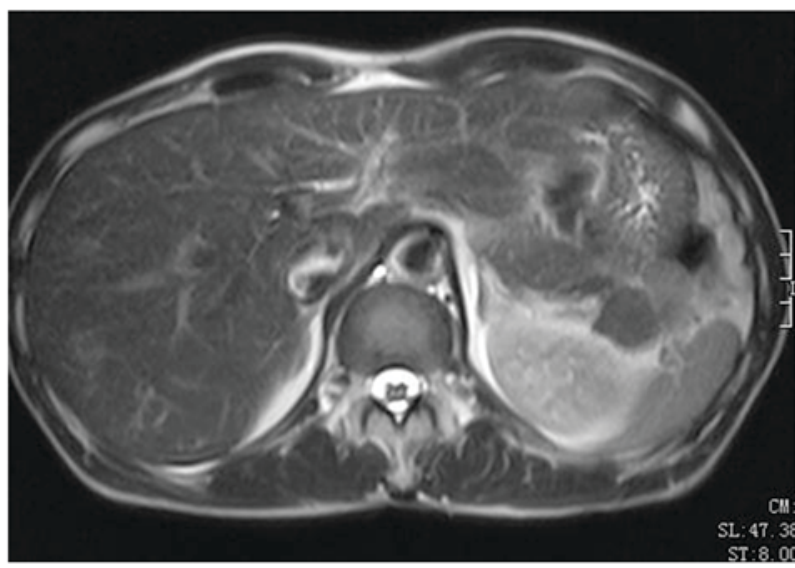

Figure 5. T2-weighted image of the lesion in the tail of the pancreas, demonstrating slight hyperintensity.

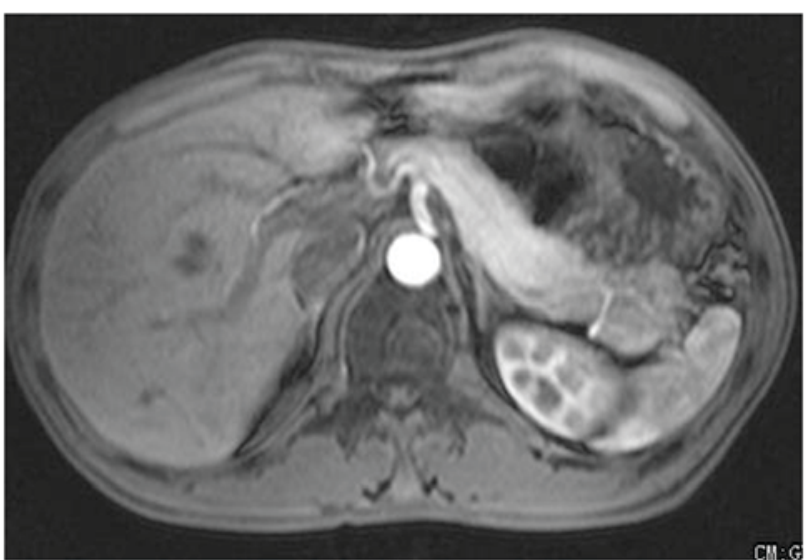

Figure 6. Lesion located in the tail of the pancreas exhibited inhomogeneous, mild enhancement in the arterial phase.

were subsequently stained with hematoxylin and eosin. The tumor was composed of bundles of staggered and spindle cells with necrosis. Atypia and mitosis were easily observable. For immunohistochemistry, paraffin-embedded sections were dewaxed, rehydrated and incubated with the following primary antibodies for $1 \mathrm{~h}$ at $37^{\circ} \mathrm{C}$ : Anti-vimentin (catalog 


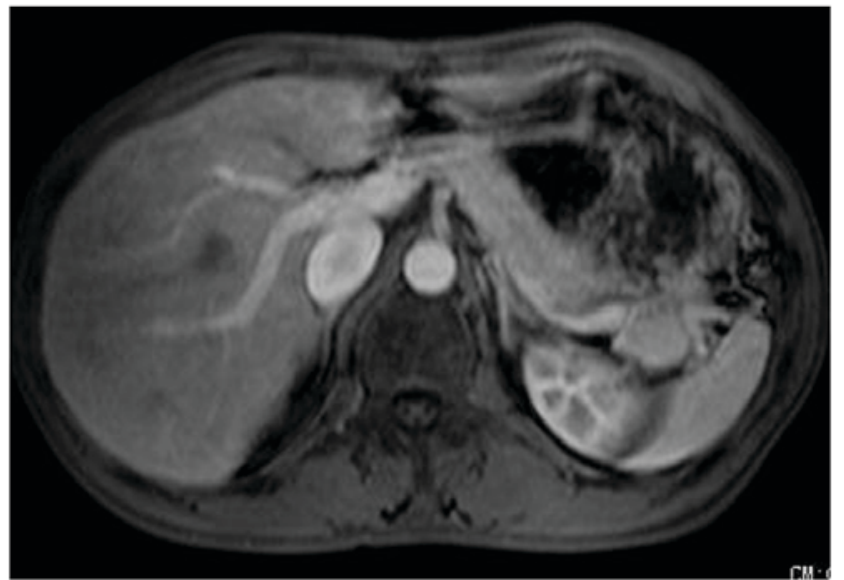

Figure 7. In the portal phase, the degree of enhancement of the lesion located in the tail of the pancreas was higher compared with the arterial phase.

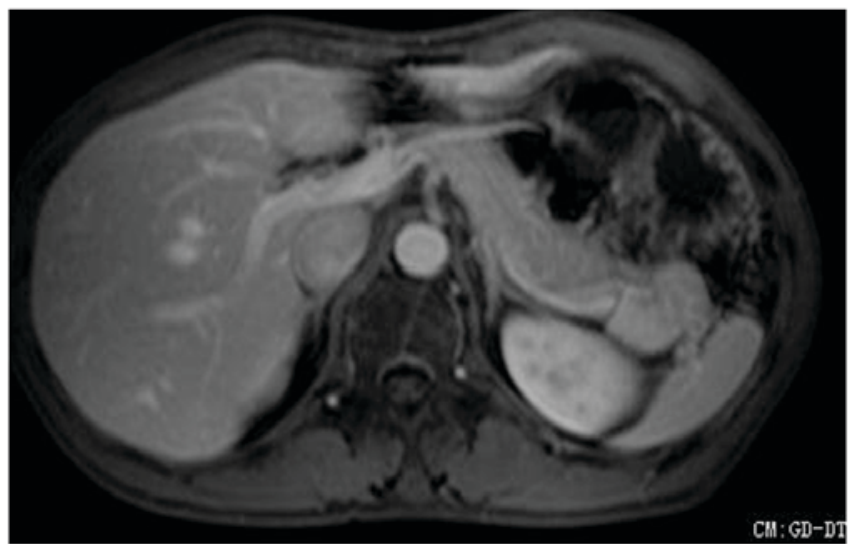

Figure 8. In the delayed phase, the lesion located in the tail of the pancreas exhibited inhomogeneous moderate enhancement.

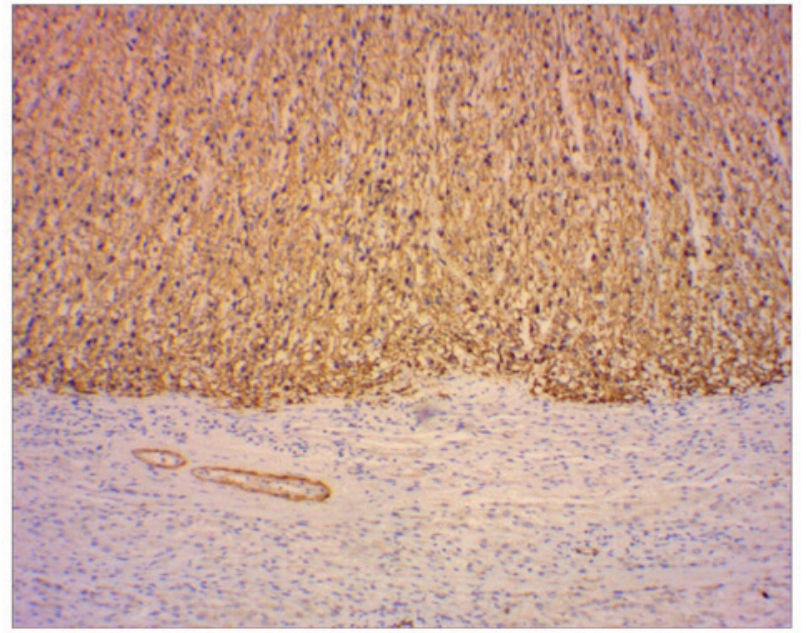

Figure 9. Cells in the mass in the liver were strongly positive for $\mathrm{H}$-caldesmon (magnification, x2.5).

no. RMA-0547; no dilution), anti-smooth muscle actin (catalog no. Kit-0006; no dilution), anti-desmin (catalog no. Kit-0023; no dilution), anti-H-caldesmon (catalog no. MAB-0634; no dilution) and anti-Ki-67 (catalog no. Kit-0005; no dilution;

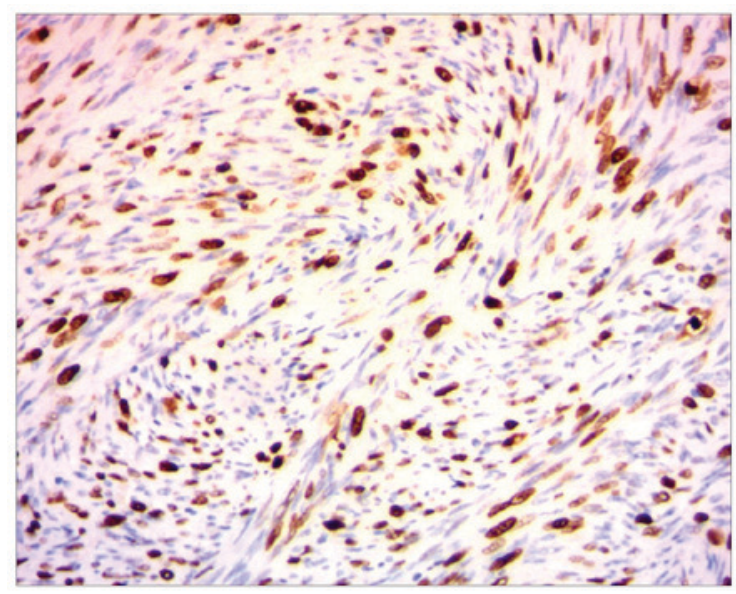

Figure 10. Cells within the mass in the splenic hilum were positive for Ki-67 (magnification, x10).

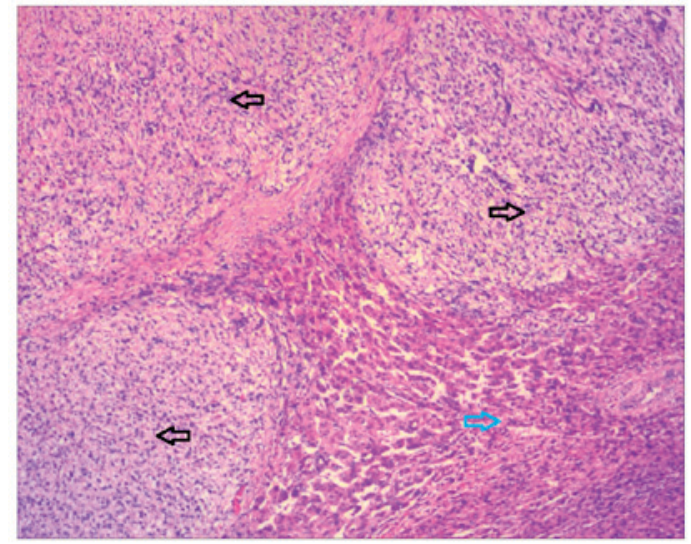

Figure 11. Mass in the liver showing tumor tissues (black arrow) and normal liver tissues (blue arrow) (magnification, x2.5).

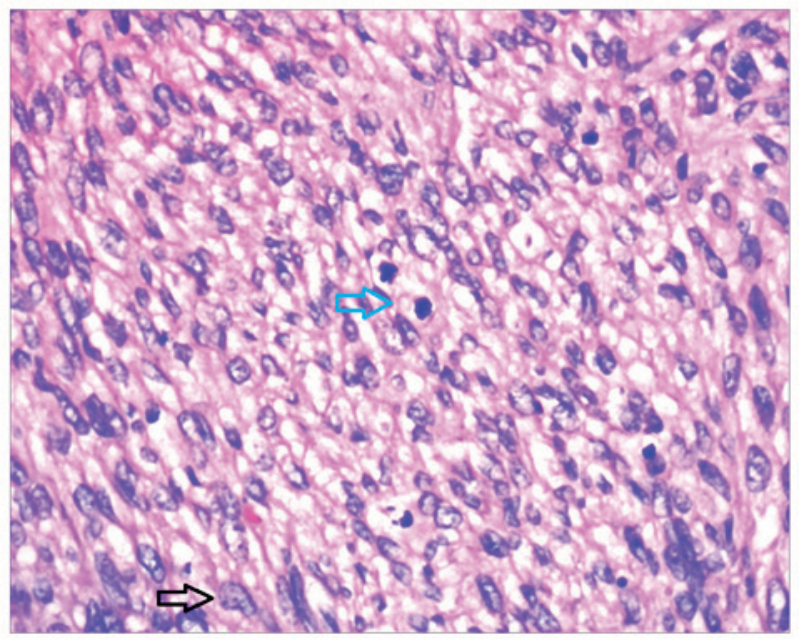

Figure 12. Mass in the splenic hilum showing a large number of spindle tumor cells (black arrow) and a mitotic figure (blue arrow; magnification, x20).

Fuzhou Maixin Biotech. Co., Ltd., Fuzhou, China). The sections were subsequently incubated with the biotin-labeled secondary antibody (catalog no., Kit-9730; no dilution; Fuzhou Maixin Biotech. Co., Ltd.) for $0.5 \mathrm{~h}$ at $37^{\circ} \mathrm{C}$. 
Immunohistochemistry demonstrated positive expression of vimentin, smooth muscle actin, desmin, H-caldesmon, Ki-67 and cluster of differentiation 34 (Figs. 9 and 10). Finally, histopathological examinations confirmed both tumors were intermediate-grade LMSs (Figs. 11 and 12).

\section{Discussion}

LMSs are rare, malignant, mesenchymal tumors derived from smooth muscle that usually appear in the retroperitoneum space behind the abdominal cavity or the uterus (3). LMSs may occur intra-abdominally in the retroperitoneum, mesentery or omentum (40-45\%), in subcutaneous or deep soft tissue of the limbs (20-30\%), in the arrector pili muscle of the skin (15-20\%), or in blood vessel walls (5\%) (1). LMSs are rare and aggressive neoplasms that arise from the blood vessel walls, and vascular LMSs occur more commonly in veins (3). Previous studies have demonstrated that vascular LMSs occur in veins five times more often than in arteries (3). The inferior vena cava locates $50 \%$ of large-vessel LMSs and the retroperitoneal veins close to the inferior vena cava, including the iliac, renal, and spermatic and ovarian veins, may be additional sites of occurrence (7). Cases of visceral vein LMSs also exist, however, those derived from the splenic vein are exceedingly rare with only a few previously documented cases $(1,2,8)$.

Venous LMSs predominately occur in middle-aged women in the fifth and sixth decades of life, and usually arise from the inferior vena cava or large veins in the lower extremities (4). Clinical manifestations depend on the tumor position in the vessel wall, venous obstruction and compression of the surrounding organs by the mass, including palpable abdominal masses and symptoms associated with thrombosis or embolism of the splenic vein, such as abdominal pain, nausea and fever $(9,10)$. Sarcomas of the great vessels are classified into luminal and mural tumors according to their site of origin (5). Luminal sarcomas of the blood vessels are more common in arteries than veins and they are characterized by rapid growth and the earlier onset of distant metastases due to intraluminal growth $(5,11)$. By contrast, mural sarcomas of the blood vessels are more common in veins, and unlike luminal sarcomas, they have more positive prognosis due to their slow and extraluminal growth (11). With regard to treatment, the majority of soft-tissue sarcomas undergo surgical resection for localized primary tumors; therefore resection offers the only opportunity for complete cure in the absence of disseminated disease. Additionally, tumor extent and histological examination to determine classification and grade is also important in guiding adjunct treatment.

Radiotherapy has been considered for the treatment of high-grade soft tissue sarcomas located in the extremities, intermediate-grade tumors of the limbs with close or positive histological margins and as a treatment for recurring low-grade sarcomas. Chemotherapy may be administered when systemic control is the primary therapeutic aim, however, the sensitivity of sarcomas, including LMSs, to chemotherapy appears to be poor (12). Therefore, as a form of adjuvant treatment for LMS, chemotherapy does not produce an evident increase in patient survival (12). There is a limited amount of evidence demonstrating increased survival following adjuvant radiation, combination radiation and chemotherapy, in addition to surgical resection. However, whether these treatment modalities should be incorporated in the general treatment of LMS is still being debated.

The current study describes a case of LMS derived from the splenic vein with concurrent liver metastasis. Histopathological examination confirmed the lesions to be intermediate-grade LMSs. An association between the masses in the spleen, splenic hilum and splenic vein was confirmed; however, the distal pancreas parenchyma did not appear to be involved. The patient underwent splenic pedicle tumor resection, splenectomy and liver tumor resection with no postoperative complications. Further information regarding patient prognosis is unavailable, as the patient did not undergo further adjuvant therapy or follow-up. LMSs of splenic vein origin are extremely rare; therefore, unique treatment recommendations or outcome data for such lesions has not been established. General management principles should follow established guidelines for other vascular LMSs and LMSs in general.

In conclusion, LMS of the splenic vein is extremely rare, and occurs predominantly in middle-aged individuals. There have not been enough documented cases to establish optimum treatment guidelines to improve patient prognosis in the shortand long-term. Therefore, the aforementioned case may enable practitioners to avoid the misdiagnosis of such patients in the future.

\section{References}

1. Niver BE, Megibowa AJ, Faust MJ and Rosenkrantz AB: Multidetector CT appearance of leiomyosarcoma of the splenic vein. Clin Radiol 66: 688-690, 2011.

2. Gage MJ, Newman E, Maldonado TS and Hajdu CH: Leiomyosarcoma of the splenic vein. J Vasc Surg 55: 1485-1487, 2012.

3. Weiss SW and Goldblum JR: Leiomyosarcoma. In: Enzinger and Weiss's Soft Tissue Tumors. 4th edition. Mosby, St. Louis, MO, 2001.

4. Tilkorn DJ, Hauser J, Ring A, Goertz O, Stricker I, Steinau HU and Kuhnen C: Leiomyosarcoma of intravascular origin-a rare tumor entity: Clinical pathological study of twelve cases. World J Surg Oncol 8: 103, 2010.

5. Burke AP and Virmani R: Sarcomas of the great vessels. A clinicopathologic study. Cancer 71: 1761-1773, 1993

6. Hines OJ, Nelson S, Quinones-Baldrich WJ and Eilber FR: Leiomyosarcoma of the inferior vena cava: Prognosis and comparison with leiomyosarcoma of other anatomic sites. Cancer 85: 1077-1083, 1999.

7. Killoran TP, Wells WA, Barth RJ and Goodwin DW: Leiomyosarcoma of the popliteal vein. Skeletal Radiol 32: 174-178, 2003.

8. Fletcher CDM: Diagnostic Histopathology of Tumors. 3rd edition. Churchill Livingstone Elsevier, Philadelphia, PA, 2007.

9. Tilkorn DJ, Lehnhardt M, Hauser J, Daigeler A, Hebebrand D, Mentzel T, Steinau HU and Kuhnen C: Intravascular leiomyosarcoma of the brachiocephalic region-report of an unusual tumour localisation: Case report and review of the literature. World J Surg Oncol 6: 113, 2008.

10. Subramaniam MM, Martinez-Rodriguez M, Navarro S, Rosaleny JG and Bosch AL: Primary intravascular myxoid leiomyosarcoma of the femoral vein presenting clinically as deep vein thrombosis: A case report. Virchows Arch 450: 235-237, 2007.

11. Székely E, Kulka J, Miklós I and Kaliszky P: Leiomyosarcomas of great vessels. Pathol Oncol Res 6: 233-236, 2000.

12. Clark MA, Fisher C, Judson I and Thomas JM: Soft-tissue sarcomas in adults. N Engl J Med 353: 701-711, 2005. 